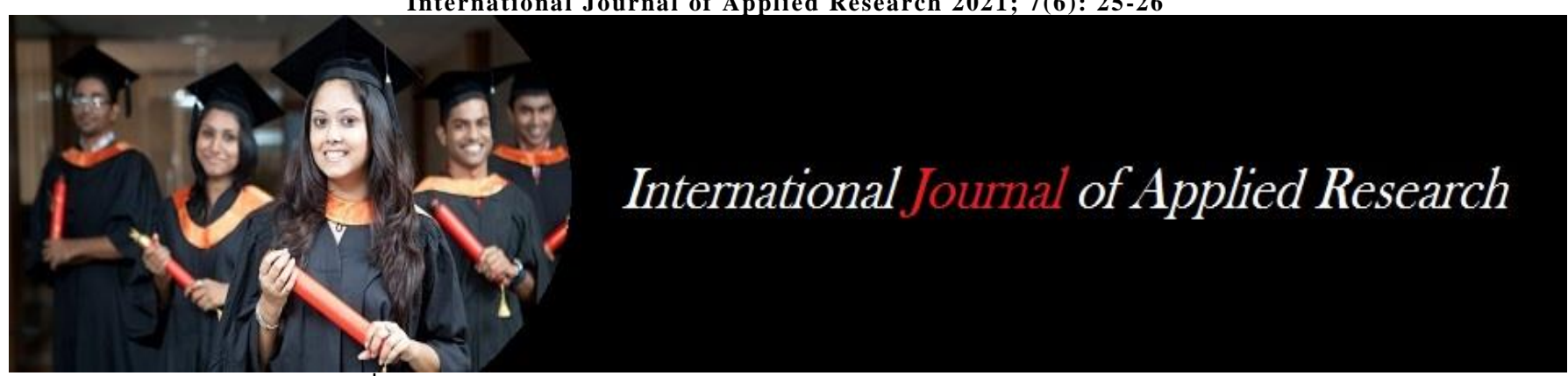

ISSN Print: 2394-7500

ISSN Online: 2394-5869

Impact Factor: 8.4

IJAR 2021; 7(6): 25-26

www.allresearchjournal.com

Received: 17-04-2021

Accepted: 21-05-2021

Dr. Nikhil Sonthalia

1st Year Resident, Department

of Medicine, КРСМСH,

Kolkata, West Bengal, India

Dr. Suman Sarkar

2nd year Resident,

Department of Medicine

KPCMCH, Kolkata, West

Bengal, India

Dr. Ankan Pathak

3rd year Resident in the

Department of Medicine

KPCMCH, Kolkata, West

Bengal, India

Dr. Nirmalya Roy

Professor, Department of

Medicine, KPCMCH, Kolkata,

West Bengal, India

Dr. Upal Sengupta

Assistant Professor,

Department of Nephrology,

KPCMCH, Kolkata, West

Bengal, India

Dr. Avinandan Banerjee

Assistant Professor,

Department of Nephrology

KPCMCH, West Bengal, India
Corresponding Author:

Dr. Suman Sarkar

2nd year Resident,

Department of Medicine

KPCMCH, Kolkata, West

Bengal, India

\section{A curious case of facial swelling}

Dr. Nikhil Sonthalia, Dr. Suman Sarkar, Dr. Ankan Pathak, Dr. Nirmalya Roy, Dr. Upal Sengupta and Dr. Avinandan Banerjee

\author{
DOI: https://doi.org/10.22271/allresearch.2021.v7.i6a.8621
}

\section{Abstract}

We present our case of a 16 year old boy presenting to us with shortness of breath and having features of both nephritic and nephrotic syndrome leading to intubation and ventilation due to pulmonary embolism. Nephrotic syndrome is characterised by heavy proteinuria $(>3 \mathrm{~g} / 24 \mathrm{hrs})$, hypertension, hypercholesterolemia, hypoalbuminemia, anasarca and microscopic hematuria whereas nephritic syndrome is characterised by the presence of proteinuria $(1-2 \mathrm{~g} / 24 \mathrm{hrs})$, hematuria, pyuria, hypertension, red blood cell casts, fluid retention, rise in serum creatinine with reduction in glomerular filtration rate. Both the diseases are separate entities however in certain cases, there can be overlapping of the components of both this syndrome.

Keywords: Curious, facial, swelling, Nephrotic, syndrome

\section{Introduction}

Glomerulonephritis is a term used to describe varying degrees of glomerular injury associated with an activated inflammatory process. It also predisposed to infection, deranged lipid profile and thrombotic states. Diffuse proliferative glomerulonephritis (DPGN) is a common and serious histological form of renal injury seen commonly in those suffering from autoimmune diseases. It advances into rapidly progressive glomerulonephritis with crescents at an accelerated rate and hence if not timely, diagnosed and treated can end up as end-stagerenal disease ${ }^{[2]}$.

The typical presentation of glomerulonephritis is insidious onset of hematuria, pyuria, hypertension, fluid retention and proteinuria. Unlike nephritic syndrome, nephrotic syndrome is characterised by massive proteinuria, anasarca, hyperlipidemia and hypoalbuminemia ${ }^{[3]}$. In some cases, however there can be features of both and when this occurs the glomerular lesion is principally in the mesangium and the membrane ${ }^{[4]}$.

\section{Case Report}

A 16 year old boy was admitted to the ICU with history of generalised body swelling for past 3 months associated with decrease in urine output and shortness of breath and puffiness of face for past 3-4 days. Initially he was brought to the emergency room in gasping condition and had to be ventilated and intubated. His calves had evidence of non-pitting edema with warmth. Well's score for pulmonary embolism showed high risk. He was therefore started on heparin. The swelling was insidious in onset and first started from the face progressing to legs and finally abdomen. 3-4 days before admission, his parents noted decrease in urine output without change in urine colour. There was no history of fever, sore throat, joint pain, loin pain, burning micturition, blood spitting or any skin lesion. He is a known case of primary hypothyroidism. He denied any addiction history.

Physical examination revealed moon facies with anasarca, blood pressure of 160/100 mm of $\mathrm{Hg}$, pulse rate of 120/min regular, falling oxygen saturation, bilateral basal crepitations with raised JVP, distended abdomen with shifting dullness, normal heart sounds with no added sounds, no pericardial rub. Scrotal swelling was present with renal angle non tender, no phimosis, no renal artery bruit. 
Blood picture showed moderate anaemia with raised total count, low albumin, increasing urea and creatinine, deranged lipid profile. Urinalysis revealed protein $2+$ along with plenty of RBC's. 24 hours urinary protein value was 6.5 gms. Ultrasound of abdomen showed raised cortical echogenicity and massive ascites. Blood and urine culture showed no growth of bacteria. C-ANCA, P-ANCA, ASO titre, anti-GBM, ANA (HEP-2), viral serology all came out to be negative. $\mathrm{C} 3$ and $\mathrm{C} 4$ complements were in low normal range.

He was therefore managed as a case of nephrotic-nephritic spectrum disorder and was started on intravenous diuretics infusion (frusemide), hydralazine-nitrates, cilnidipine, telmisartan, prazosin, anticoagulants (heparin shifted to rivaroxaban), thyroxine and statins. His edema started reducing by 6th day, blood pressure was controlled after 10 th day and by 14 th day his urine showed protein of $1+$

A kidney biopsy was done with DIF which revealed diffuse proliferative glomerulonephritis with immune complex deposits probably infection related glomerulonephritis (IRGN). He was there after started on steroids and mycophenolate mofetil.

\section{Discussion}

C3 glomerulonephritis is a rare form of glomerulonephritis that affects both adults and children. It results from result from abnormal regulation of the alternative complement pathway. The diagnosis is made with the help of a kidney biopsy along with immunofluorescence staining and complement studies. Immunofluorescence microscopy almost always shows deposits of C3 along the glomerular, tubular and Bowman's capsule basement membranes, as well as mesangial rings ${ }^{[5]}$.

A renal biopsy is generally not indicated for the diagnosis of nephritic syndrome but it is sometimes necessary when there is an overlap of features of both nephrotic and nephritic disorders. In view of the features consistent with nephrotic syndrome, it was plausible to carry out kidney biopsy to delineate the histological type. Also renal biopsy is required to differentiate between nephritic syndrome and nephrotic, the latter requiring steroids to see whether it is steroid responsive or steroid resistant ${ }^{[6]}$.

\section{Conclusion}

As steroids use in nephritic disorders is not clear, it should not be started in cases of nephrotic-nephritic overlap disorder until and unless a renal biopsy has been performed which is necessary to differentiate between the two. In some situations where it is not possible to perform a renal biopsy, it is better to treat the case as nephritic type and wait for the progression of the disease.

\section{References}

1. Adedoyin OT et al. Acute glomerulonephritis mimicking nephrotic syndrome. Niger $J$ Paediatr 2016;43(2):95-98.

DOI:http://dx.doi.org/10.4314/njp.v43i2.6

2. Ikhlas M, Anjum F. Diffuse Proliferative Glomerulonephritis. [Updated 2020 Dec 4]. In: StatPearls [Internet]. Treasure Island (FL): StatPearls Publishing, 2021.

3. Eison TM, Ault BH, Jones DP, Chesney RW, Wyatt RJ. Poststreptococcal acute glomerulonephritis in children: Clinical features and pathogenesis. Pediatr. Nephrol 2011;26:165-180.

4. Alpers C. The Kidney. In: Kumar V, Abbas AK, Fausto N, Aster J, editors. Robbins \& Cotran Pathologic Basis of Disease. 7. Saunders Elsevier, 2012, pp. 982-983.
5. Pickering MC, D'Agati VD, Nester CM, et al. C3 glomerulopathy: consensusreport. Kidney Int 2013;84:1079.

6. Tsu Jung Yang et al. Role of Steroids in Poststreptococcal Glomerulonephritis Without Crescents on Renal Biopsy Cureus 2018;10(8):e3150. Published online 2018 Aug 15. doi: 10.7759/cureus.3150 